\title{
Analysis of a Budding Yeast Cell Cycle Model Using the Shapes of Local Sensitivity Functions
}

\author{
A. LOVRICS, ${ }^{1}$ I. GY. ZSÉLY, ${ }^{1}$ A. CSIKÁSZ-NAGY, ${ }^{2}$ J. ZÁDOR, ${ }^{1}$ T. TURÁNYI, ${ }^{1}$ B. NOVÁKK ${ }^{3,4}$ \\ ${ }^{1}$ Laboratory for Chemical Kinetics, Institute of Chemistry, Eötvös University (ELTE), Budapest, Hungary \\ ${ }^{2}$ Microsoft Research-University of Trento, Centre for Computational and Systems Biology, Trento, Italy \\ ${ }^{3}$ Molecular Network Dynamics Research Group, Budapest University of Technology and Economics (BUTE), \\ Budapest, Hungary \\ ${ }^{4}$ Integrative Systems Biology, Department of Biochemistry, Oxford University, Oxford, UK
}

Received 21 November 2007; revised 29 April 2008; accepted 30 April 2008

DOI 10.1002/kin.20366

Published online in Wiley InterScience (www.interscience.wiley.com).

\begin{abstract}
The Chen et al. (Mol Biol Cell 2000, 11, 369-391) budding yeast cell cycle model is a biochemical kinetic model that describes how the controlling protein concentrations change during a proliferation cycle. Time dependence of local sensitivity coefficients was calculated for all variables and parameters of the model. Some of the local sensitivity coefficients-time functions could also be obtained from another one by multiplying it with a constant, which means that these functions exhibit global similarity. Local similarity of the sensitivity functions was also detected. The distance of the shapes of two scaled sensitivity functions was defined by the integrated squared difference of these functions. The distance matrices of function shapes were interpreted by a clustering method, and the shapes could be sorted to two main groups for each model variable. The presence of the global similarity of sensitivity functions means that the change of some enzyme activities can be fully compensated by changing the activity of other enzymes. This feature can be related to the robustness of living organisms. (c) 2008 Wiley Periodicals, Inc. Int J Chem Kinet 40: 710-720, 2008
\end{abstract}

Correspondence to: T. Turányi; e-mail: turanyi@ chem.elte.hu. Present address of A. Lovrics: School of Mathematical Sciences, University of Nottingham, Nottingham, UK.

Present address of J. Zádor: Combustion Research Facility, Sandia National Laboratories, Livermore, CA.

Contract grant sponsor: OTKA.

Contract grant numbers: T68256 and F60414.

(c) 2008 Wiley Periodicals, Inc.

\section{INTRODUCTION}

The cell cycle control is the best known in the budding yeast (Saccharomyces cerevisiae) among eukaryotic organisms. This control is based on the role of Cdk (cyclin-dependent protein kinase) molecules in coordinating the events of DNA synthesis, bud emergence, spindle formation, nuclear division, and cell separation. After the cell division, for a long period only the 
cell size increases (phase G1) followed by the DNA synthesis (phase S/G2). Finally, two identical nuclei and then two daughter cells are formed (mitotic phase $\mathrm{M}$ of the cell cycle). A series of mathematical models, using sets of differential and algebraic equations, have been created to describe the change of enzyme concentrations during a cell cycle in wild-type cells and in various mutants [1-4].

The cell cycle model investigated here was created by Chen et al. in 2000 [3]. The Chen et al. model is defined by a 13-variable set of ordinary differential equations and related algebraic equations. This model includes 73 parameters and contains all the basic features of the Cdk's control.

Timescale and dimension analyses of this cell cycle model have been carried out by the authors [5]. The analyses were based on the investigation of the Jacobian of the set of differential equations. In the model, the cell mass is exponentially growing, which causes a single constant positive eigenvalue of the Jacobian. All other eigenvalues are related to chemical processes, and these are discussed in the rest of the paper. If at any time the largest eigenvalue is positive, then small concentration differences can be amplified and the system is in an excitation period. If the largest eigenvalue is negative, in most cases the concentration differences tend to be damped and the system is in a relaxation period.

The analysis revealed [5] the presence of an excitation period (labeled E4a) just before the cell division. The next excitation period (E4b) occurs just after the cell division. At the end of phase G1, there is an excitation period E1. In phase $S$ of the cell cycle, two more excitation periods are located. The very strong excitation period E3 is embedded into the period of weaker excitation E2. The biochemical origin of all these excitation periods is discussed in detail in [5].

The change of the dynamical dimension during the cell cycle was also investigated. The dynamical dimension rises up to seven in the excitation periods and decreases to one in each relaxation period [5]. This means that in some periods the original model of 13 variables could be replaced by a seven-variate system of ordinary differential equations (ODEs), whereas in other periods all concentrations are in steady state, and their change is dictated by the increase of the cell mass only.

In this paper, the Chen model [3] of the cell cycle of budding yeast is investigated further using local sensitivity analysis, and the new results are compared with our previous ones on timescale and dimension analyses.

\section{SENSITIVITY ANALYSIS OF DYNAMICAL MODELS}

Sensitivity analysis is the name of a family of mathematical methods that investigate the relation between the parameters and the output of models. Comprehensive reviews of the various methods of sensitivity analysis were recently published by Saltelli et al. [6,7]. Applications of sensitivity analysis in reaction kinetics were discussed by Turányi [8] and Tomlin et al. [9]. Utilization of sensitivity analysis is widespread in combustion and atmospheric chemistry, but fewer and mainly recent applications of these methods can be found in the investigation of biochemical kinetic systems (see, e.g., [10-12]).

A dynamical model can be characterized by the following initial value problem:

$$
\mathrm{d} \mathrm{Y} / \mathrm{d} t=\mathbf{f}(\mathbf{Y}, \mathbf{p}), \quad \mathbf{Y}(0)=\mathbf{Y}^{0}
$$

where $t$ is the time, $\mathbf{Y}$ is the $n$-vector of variables, $\mathbf{p}$ is the $m$-vector of parameters, $\mathbf{Y}^{0}$ is the vector of the initial values of the variables, and $\mathbf{f}$ is the right-hand side of the differential equations.

The local sensitivity function $s_{i k}(t)$ can be calculated (see, e.g., [8]) by solving the following initial value problem:

$$
\dot{\mathbf{S}}=\mathbf{J S}+\mathbf{F} \quad \mathbf{S}(0)=\mathbf{0}
$$

where $\mathbf{S}(t)=\left\{s_{i k}(t)\right\}=\left\{\partial Y_{i} / \partial p_{k}\right\}$ is the timedependent local sensitivity matrix, $\mathbf{J}$ is the Jacobian $\left(\mathbf{J}=\left\{\partial f_{i} / \partial Y_{j}\right\}\right)$, and matrix $\mathbf{F}$ contains the derivatives of the right-hand side of the ODE with respect to the parameters $\left(\mathbf{F}=\left\{\partial f_{i} / \partial p_{k}\right\}\right)$. The $s_{i k}(t)$ local sensitivity functions show the effect of a small perturbation of parameter $k$ on the change of variable $i$ at time $t$. Sensitivity coefficients form the sensitivity matrix: $\mathbf{S}$ $=\left\{\partial Y_{i} / \partial p_{k}\right\}$. In the case of a general mathematical model, no relation is expected among the rows and/or the columns of the sensitivity matrix. However, in several chemical kinetic systems the following relations have been observed:

(i) Local similarity: Value $\lambda_{i j}(t)$ depends on time $t$ and the model results $Y_{i}$ and $Y_{j}$ selected, but is independent of the parameter $p_{k}$ perturbed.

$$
\lambda_{i j}(t)=\frac{s_{i k}(t)}{s_{j k}(t)}
$$

(ii) Scaling relation: Equation (4) is valid for any parameter $p_{k}$. Existence of a scaling relation 
includes the presence of local similarity.

$$
\frac{\mathrm{d} Y_{i} / \mathrm{d} t}{\mathrm{~d} Y_{j} / \mathrm{d} t}=\frac{s_{i k}(t)}{s_{j k}(t)}
$$

(iii) Global similarity: Value $\mu_{i k m}$ is independent of $t$ (within an interval).

$$
\mu_{i k m}=\frac{s_{i k}(t)}{s_{i m}(t)}
$$

Global similarity and in some cases scaling relation of sensitivity functions were detected by Rabitz et al. in several flame models $[13,14]$ and in thermal runaway systems [15].

In a series of works, Zsély et al. [16-19] presented new results on the similarity of sensitivity functions in chemical kinetic models. They have shown that the existence of low-dimensional slow manifolds in chemical kinetic systems may cause local similarity. Global similarity emerges if local similarity is present and the sensitivity differential equations are pseudohomogeneous. The latter means that in Eq. (2) relation $\|\mathbf{F}\| \ll\|\mathbf{J S}\|$ holds $[15,16]$. Pseudohomogeneity is related to the existence of excitation periods, like the autocatalytic runaway during chain-branching explosions.

Zádor et al. [18] have shown that the level of local similarity can be characterized by angle $\theta$ of the directions of two sensitivity vectors in the space of parameters, which can be calculated in the following way:

$$
\hat{\mathbf{s}}_{i}^{\mathrm{T}} \hat{\mathbf{s}}_{j}=\cos \theta_{i j}
$$

Here $\hat{\mathbf{s}}_{i}=\mathbf{s}_{i} /\left\|\mathbf{s}_{i}\right\|$ and $\hat{\mathbf{s}}_{j}=\mathbf{s}_{j} /\left\|\mathbf{s}_{j}\right\|$ are unit length sensitivity vectors. Measure $\cos \theta_{i j}=+1$ or $\cos \theta_{i j}=-1$ indicates that the sensitivity vectors are correlated and therefore are locally similar.

Since in the cell cycle model of Chen et al. [3] we had detected [5] low-dimensional manifolds (even dimension one) and excitation periods, we expected that the local sensitivity functions of the model would exhibit global similarity. Therefore, a local sensitivity analysis of the cell cycle model was carried out.

\section{SENSITIVITY ANALYSIS OF THE CELL CYCLE MODEL}

Local sensitivity functions $s_{i j}(t)=\left(\partial Y_{i}(t) / \partial p_{j}\right)$ of the Chen et al. [3] model were calculated for all variables and parameters of the model. Integration of the differential equations and calculation of sensitivities were carried out by subroutine DASAC [20] that had been distributed with the SENKIN code [21] of the CHEMKIN-II package [22].

To compensate the effect of very different parameter values, the calculated sensitivity functions were seminormalized: $s_{i j}(t)=p_{j}\left(\partial Y_{i}(t) / \partial p_{j}\right)=$ $\left(\partial Y_{i}(t) / \partial \ln p_{j}\right)$, and in all further discussions these $\left(\partial Y_{i}(t) / \partial \ln p_{j}\right)$ functions are referred to as sensitivity functions. The initial values of all sensitivities are zero; therefore, the sensitivity functions were different in the first cycle compared to the later cycles, but from the second cell division these became periodic functions. In the Chen model, the period of the cell cycle depends only on the value of nutrition parameter $m u$, therefore the sensitivity functions of each parameter except for $m u$ became periodic after a transition time. In this paper, sensitivity functions of the fourth cycle were investigated and plotted, which were numerically identical to the sensitivity functions calculated for the later cycles. Sensitivity functions of $m u$ were not really periodic, but changed little from one cycle to the other, and therefore the corresponding sensitivity functions were not excluded from the function shape investigations. Sensitivity vectors of variables mass and Vi20 were not considered, because the values of these variables depend on few parameters only and almost all elements of the sensitivity vectors are zero. Sensitivities of parameters $f$, thres 1 , thres were also excluded from the investigations. These parameters are not related to chemical reactions and have special role in the model. Parameter $f$ is an experimentally determined value, indicating the ratio of the masses of the two newly formed cells. Parameters thres 1 and thres are threshold values and are not directly related to physical parameters.

Figures 1,2, and 3 show the sensitivity functions of $C l n 2, C l b 2 T$, and $C d c 20 T$, respectively, during a cycle. Similar figures were prepared for all other variables of the model. It is clear that the rise of the sensitivity functions in most cases coincide with the positive eigenvalue (gray) periods. Perturbing a parameter, after a delay, causes a deviation of the concentration set compared to that of the original model. In the excitable (gray) regions, the distance between the original and the perturbed concentration sets increases, and therefore the absolute value of the sensitivity functions rises. In the relaxation (white) regions, all eigenvalues are negative, therefore the distance between the two concentration sets usually decreases, and the sensitivity functions do relax toward zero.

Inspecting Figs. 1-3, it is obvious that several (but not all) sensitivity functions of a given variable can be obtained from another one by multiplying it with a constant, which means that these functions are globally similar. Also, we have found that the sensitivity functions can be sorted to several groups according to 


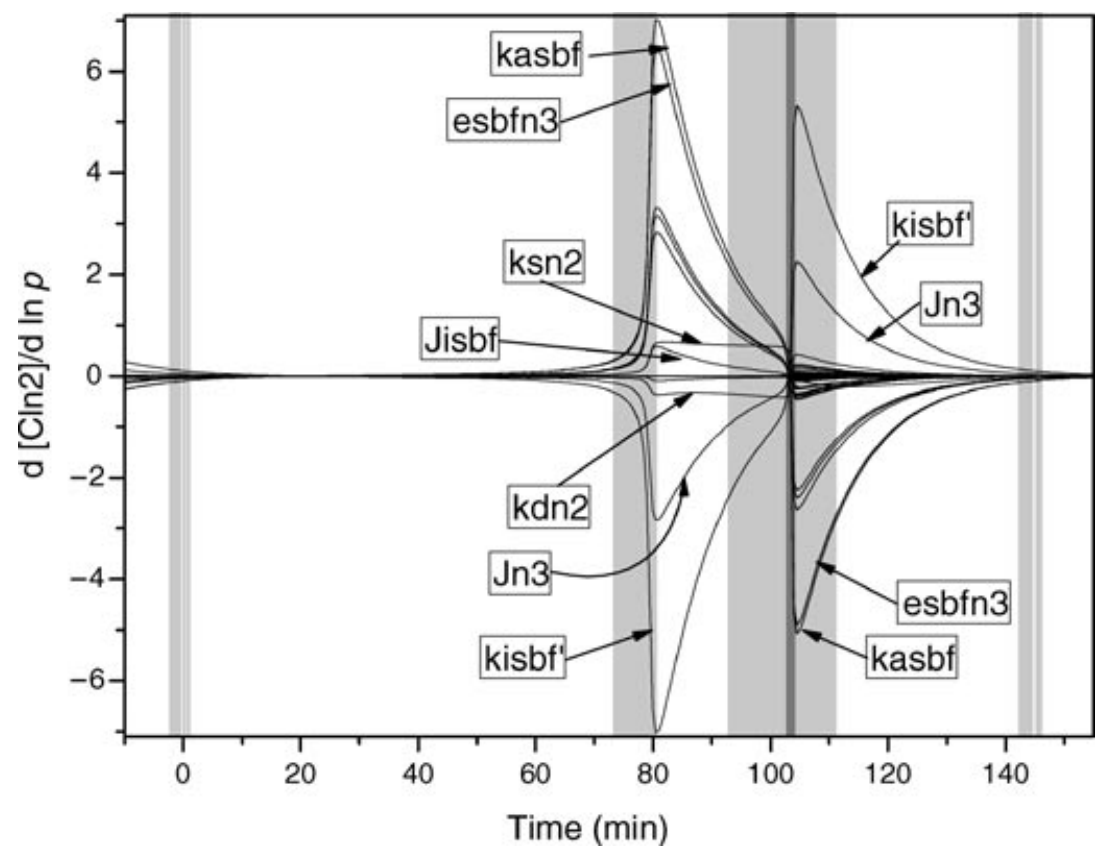

Figure 1 Sensitivity functions of the concentration of enzyme $C \ln 2$. Here and in all other figures the excitation periods are denoted by gray and strong excitation period E3 is denoted by dark gray. Time zero marks the time of cell division. The labels identify the parameters according to Chen et al. [3].

their shapes, and that some of the sensitivity functions cannot be related to others this way.

To carry out the grouping of the sensitivity functions $s_{i k}(t)$ in time interval $\left[t_{1}, t_{2}\right]$ in an objective way, their shapes were compared via the following procedure. First, the functions were scaled to unit peak value: $\widehat{s}_{i k}(t)=s_{i k}(t) / \max \left|s_{i k}(t)\right|$. Then, the integrated difference of the two-scaled sensitivity functions was arranged to matrix $\mathbf{C}$ :

$$
C_{i}(k, l)=\int_{t_{1}}^{t_{2}}\left(\widehat{s}_{i k}(t)-\widehat{s}_{i l}(t)\right)^{2} \mathrm{~d} t
$$

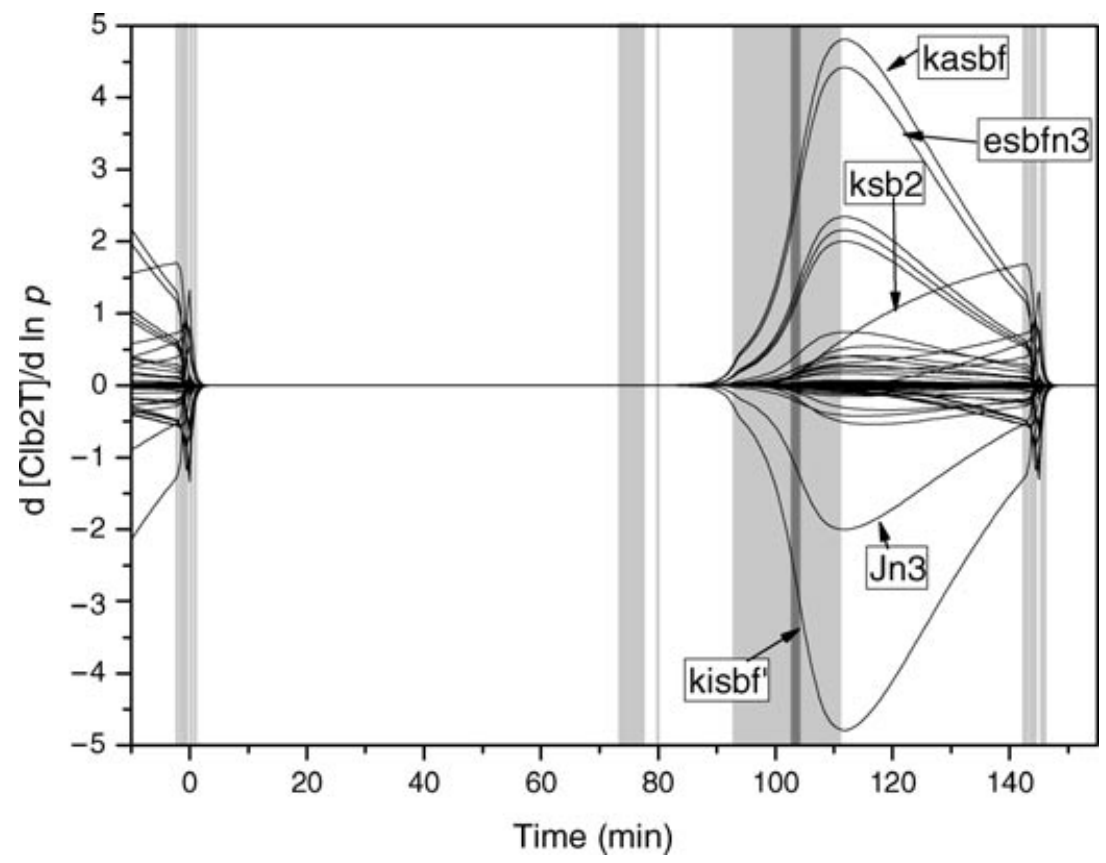

Figure 2 Sensitivity functions of the concentration of enzyme $C l b 2 T$. 


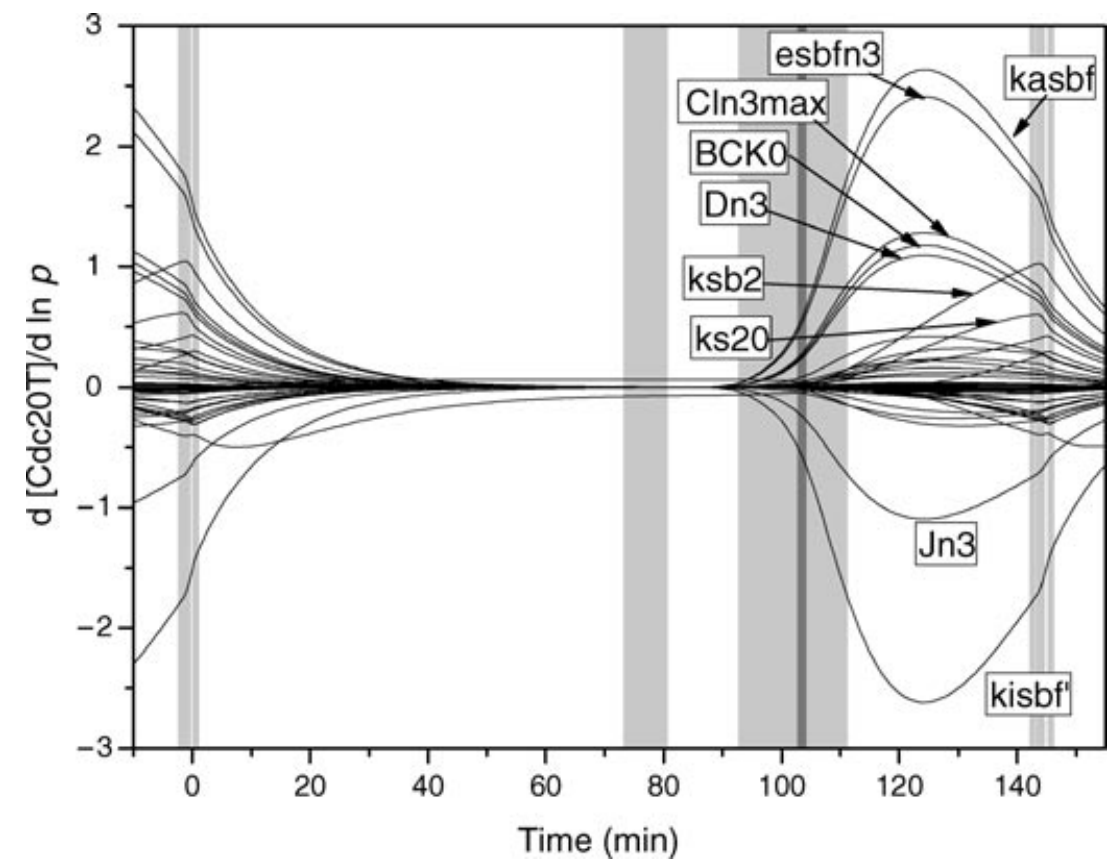

Figure 3 Sensitivity functions of the concentration of enzyme $C d c 20 T$.

This is a distance matrix of sensitivity function shapes, since if the local sensitivity functions of variable $i$ with respect to parameters $k$ and $l$ have identical shapes, then $C_{i}(k, l)$ is zero, and large $C_{i}(k, l)$ value indicates very different shapes. This distance matrix was used as an input of a clustering code.

Cluster analysis is a technique that discovers groups of objects that are similar to each other [23]. The similarity of the objects can be characterized numerically by a distance matrix. The diagonal of this matrix should be zero, and it is a symmetric matrix. The hierarchical approach of clustering was selected, because we wanted an unbiased identification of groups of similar function shapes. Agglomerative and divisive clustering are the two basic approaches of hierarchical cluster analysis. Agglomerative methods join smaller clusters into larger clusters, whereas divisive methods split larger clusters into smaller clusters. We used here the weighted average clustering method, which is an agglomerative one. This method is one of the options of Fortran code HCINFLU [24], which was applied in our calculations. The number of the identified clusters depends on a dissimilarity threshold value of the cluster analysis. When two clusters are merged, the code shows the level of dissimilarity between these two clusters. When the dissimilarity value was small, the function shapes always fitted well to one of the clusters. When there was a jump in the range of dissimilarity values and for the first time the dissimilarity value became large, two clusters of dissimilar function shapes would have been merged. The cluster analysis was always stopped before this point.

Figure 4 shows the scaled sensitivity functions of $C \ln 2$. Most of the scaled sensitivity functions of variable $C \ln 2$ follow two curves, indicated by a thick solid and a thick-dotted line in the figure. For each variable, the clustering calculations revealed that most of the sensitivity functions were of globally similar, and these functions could be sorted to two basic groups of global similarity. This is a qualitatively new feature of the global similarity of sensitivity functions that have not been observed in other chemical kinetic systems.

Table I summarizes all pieces of information for the shapes of the sensitivity functions of this cell cycle model. Identical labels in different rows of the same column of Table I means that the corresponding sensitivity functions are globally similar. Labels A and B indicate the two basic shapes for each variable. Labels $\mathrm{A} 1, \mathrm{~A} 2, \ldots$ (or $\mathrm{B} 1, \mathrm{~B} 2, \ldots$ ) indicate that the corresponding shapes are basically similar, but these functions are different in a small section. Identical shapes were denoted by exactly identical labels for each variable. Sign X shows that no similar sensitivity function was found among the sensitivity functions of the same variable.

Local similarity of the sensitivity vectors was investigated by two different methods. First at three selected times located at the ends of the main excitation periods $(0.86,80.56$, and $111.66 \mathrm{~min}$ from cell division), the ratios of the sensitivity coefficients were calculated 


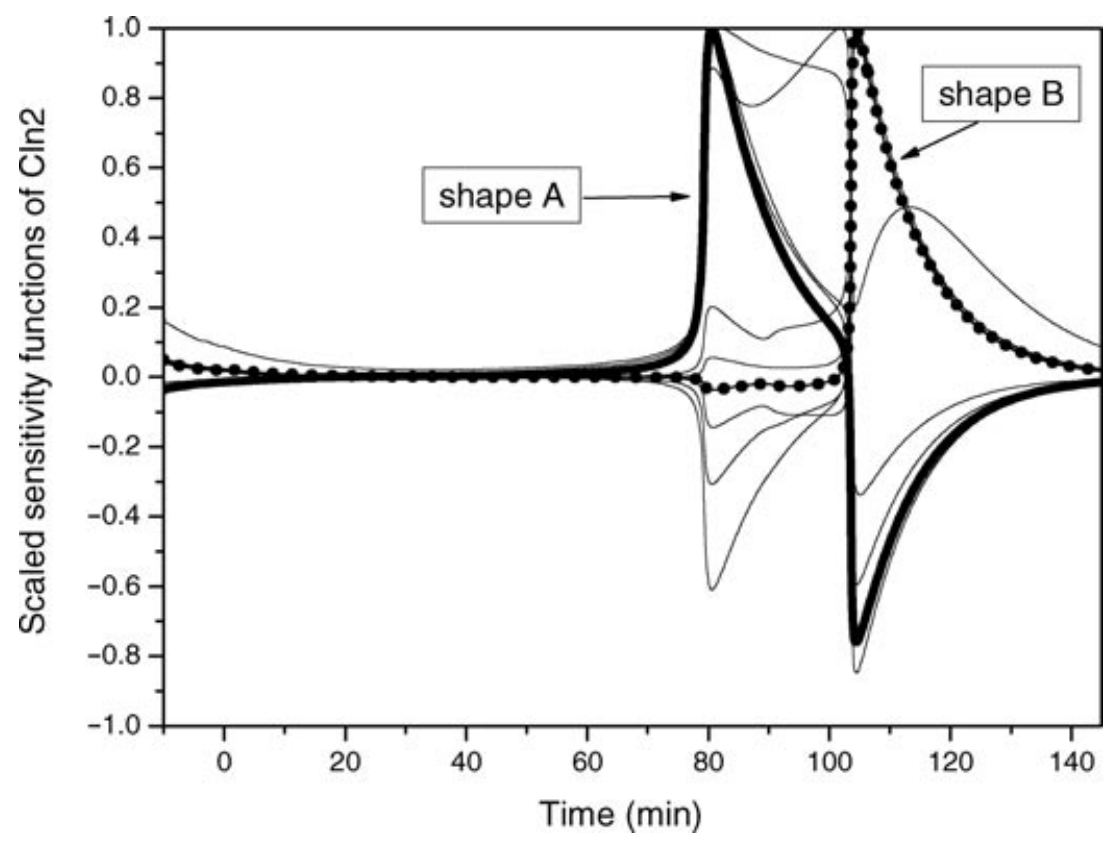

Figure 4 Scaled sensitivity functions of $C \ln 2$. The thick solid line represents 10 overlapping functions of shape A (cf. Table I), whereas the thick-dotted line represents 38 overlapping functions of shape B. There are nine scaled sensitivity functions that do not show similarity to shapes A or B.

according to Eq. (3). At a given time and pair of variables $Y_{i}$ and $Y_{j}$ those sensitivity coefficients were considered locally similar, for which the calculated ratio was identical within a tolerance margin. This way, at each reaction time 4-12 groups of locally similar sensitivity coefficients were identified to each of the possible 36 pairs of variables. A part of these groups were different at different reaction times.

We also wanted to identify whether there are local similarity relations that extend to all variables and are valid during the whole period. Therefore, as the next step, groups of sensitivity coefficients were selected using the results of the previous analysis, and the correlation of the sensitivity vectors was calculated using Eq. (6). The measure $\cos \theta_{i j}= \pm 1$ indicates that the coefficients belonging to the sensitivity vectors are locally similar. Figure 5 shows the correlation of the sensitivity vector of $C \ln 2$ with the sensitivity vectors of all other variables, considering parameters kasbf, kisbf', esbfn3, BCK0; CLN3MAX, Dn3, and Jn3. During the whole period and for each pair of variables, $\cos \theta$ is close to \pm 1 . In a similar way, local similarity that was valid during the whole period and for all pairs of variables was detected for parameters kiswi', kaswi, and Jaswi. Many other alike parameter groups were detected, but for these groups local similarity was not fulfilled in some (narrow) range of the cell cycle period or was not valid for the sensitivity functions of all pairs of variables.

Results of the analysis presented above show that in this cell cycle model multiple global similarity and multiple local similarity relations exist. In combustion models only single global similarity and single local similarity have been found $[14,16]$.

Table I The Inventory of All Sensitivity Function Shapes

\begin{tabular}{lccccccccc}
\hline & Cln2 & Clb2T & Clb5T & Sic1T & C2 & C5 & Cdc20T & Cdc20 & HCT \\
\hline BCK0 & A & A1 & A & A & A & A & A & A & A1 \\
CLN3MAX & A & A1 & A & A & A & A & A & A & A1 \\
Dn3 & A & A1 & A & A & A & A & A & A & A1 \\
kasbf & A & A1 & A & A & A & A & A & A & A1 \\
kisbf & A & A1 & A & A & A & A & A & A & A1 \\
Jn3 & A & A1 & A & A & A & A & A & A & A1 \\
Jisbf & A & A1 & A & A & A & A & A & A & A1 \\
esbfn3 & A & A1 & A & A & A & A & A & A & A1 \\
\hline
\end{tabular}


Table I (Continued)

\begin{tabular}{|c|c|c|c|c|c|c|c|c|c|}
\hline & $\mathrm{Cln} 2$ & $\mathrm{Clb} 2 \mathrm{~T}$ & Clb5T & Sic1T & $\mathrm{C} 2$ & $\mathrm{C} 5$ & Cdc20T & $\mathrm{Cdc} 20$ & HCT \\
\hline $\mathrm{mu}$ & A & A 2 & A & A & A & A & A & $X$ & A1 \\
\hline $\mathrm{ksn} 2^{\prime \prime}$ & $X$ & A2 & $X$ & A & A & A & A & A & A1 \\
\hline Jasbf & $X$ & $\mathrm{~A} 2$ & $X$ & A & A & $\mathrm{A}$ & A & A & A1 \\
\hline $\mathrm{kdn} 2$ & $X$ & $\mathrm{~A} 2$ & $X$ & A & $X$ & A & A & A & $\mathrm{A} 1$ \\
\hline $\mathrm{ksb}^{\prime \prime}$ & $X$ & $\mathrm{~A} 2$ & $X$ & $X$ & $X$ & $X$ & A & A & $\mathrm{A} 2$ \\
\hline kdib5 & $X$ & A1 & $X$ & $X$ & $X$ & $X$ & A & A & $\mathrm{A} 1$ \\
\hline kasb5 & A & $\mathrm{X}$ & A & $X$ & B1 & $\mathrm{X}$ & $X$ & $X$ & A1 \\
\hline ec $\ln 3$ & B & A1 & B & $\mathrm{X}$ & $\mathrm{X}$ & B1 & A & A & $\mathrm{A} 2$ \\
\hline ec1k2 & B & A1 & B & $X$ & $X$ & B1 & A & A & $\mathrm{A} 2$ \\
\hline $\mathrm{ksc1}^{\prime}$ & B & $\mathrm{A} 1$ & B & $X$ & $X$ & B1 & A & A & $\mathrm{A} 2$ \\
\hline kd1c1 & B & $\mathrm{A} 1$ & B & $X$ & $X$ & B1 & A & A & $\mathrm{A} 2$ \\
\hline $\mathrm{kd} 2 \mathrm{c} 1$ & B & A1 & B & $X$ & $X$ & B1 & A & A & $\mathrm{A} 2$ \\
\hline Jiswi & B & A1 & B & $X$ & B1 & B1 & A & A & $\mathrm{A} 2$ \\
\hline eit1n2 & B & A2 & B & B & $\mathrm{X}$ & B2 & A & A & A1 \\
\hline $\mathrm{ksc}^{\prime \prime}$ & B & A1 & B & $X$ & B1 & B1 & A & A & $\mathrm{A} 2$ \\
\hline kaswi & B & A1 & B & B & B1 & B1 & A & A & A2 \\
\hline kiswi' $^{\prime}$ & B & $\mathrm{A} 1$ & B & B & B1 & B1 & A & A & $\mathrm{A} 2$ \\
\hline Jaswi & B & A1 & B & B & B1 & B1 & A & A & $\mathrm{A} 2$ \\
\hline $\mathrm{ks} 20^{\prime}$ & B & A2 & $X$ & $X$ & $\mathrm{~A}$ & $X$ & $X$ & $X$ & A1 \\
\hline ksb2' & B & A 2 & B & B & $\mathrm{X}$ & $\mathrm{X}$ & A & A & $\mathrm{X}$ \\
\hline kamcm & B & A 2 & B & B & B2 & B2 & A & A & B \\
\hline kimcm & B & A2 & B & B & B2 & B2 & A & A & B \\
\hline Jamcm & B & A2 & B & B & B2 & B2 & A & A & B \\
\hline Jimcm & B & $\mathrm{A} 2$ & B & B & B2 & B2 & $\mathrm{A}$ & A & B \\
\hline $\mathrm{Jd} 2 \mathrm{c} 1$ & B & $\mathrm{A} 2$ & B & $X$ & $X$ & B1 & B1 & B & $\mathrm{A} 2$ \\
\hline ec1b5 & B & A2 & B & $X$ & $X$ & B1 & B1 & B & A2 \\
\hline kasb2 & $X$ & $\mathrm{X}$ & $X$ & $X$ & B1 & $\mathrm{X}$ & $\mathrm{X}$ & $X$ & A1 \\
\hline kdib2 & $X$ & $X$ & $X$ & $X$ & B1 & $X$ & $X$ & $X$ & A1 \\
\hline $\mathrm{ksb5}^{\prime}$ & $X$ & $X$ & $X$ & $X$ & $X$ & $X$ & B1 & B & $\mathrm{A} 2$ \\
\hline $\mathrm{kdb} 5^{\prime}$ & $X$ & $X$ & $X$ & $X$ & $X$ & $X$ & B1 & B & $\mathrm{A} 2$ \\
\hline kisbf ${ }^{\prime \prime}$ & B & $X$ & B & $X$ & $X$ & $X$ & B1 & B & $X$ \\
\hline esbfb5 & B & $X$ & B & $X$ & $X$ & $X$ & B1 & A & $X$ \\
\hline kdb5" & B & $X$ & $X$ & $X$ & B1 & $X$ & B1 & B & $\mathrm{A} 2$ \\
\hline eit1b5 & B & $X$ & B & B & B2 & B2 & B1 & B & A2 \\
\hline kiswi" & B & $X$ & $X$ & $X$ & B1 & $\mathrm{X}$ & $X$ & $X$ & B \\
\hline $\mathrm{kd} 20$ & B & B2 & $X$ & B & B3 & $X$ & $X$ & $X$ & $X$ \\
\hline ka20 & B & B1 & $X$ & $X$ & B3 & $X$ & B2 & $X$ & $X$ \\
\hline ec1b2 & B & B1 & $X$ & $X$ & $X$ & $\mathrm{X}$ & B2 & B & $X$ \\
\hline kat $^{\prime}$ & B & $\mathrm{X}$ & $X$ & B & B4 & B2 & B1 & B & $X$ \\
\hline $\mathrm{ksb} 2^{\prime \prime}$ & B & $\mathrm{X}$ & $X$ & B & B4 & B2 & B1 & B & B \\
\hline $\mathrm{kdb} 2^{\prime \prime}$ & B & B1 & $X$ & B & B5 & B2 & B2 & B & $X$ \\
\hline $\mathrm{kdb} 2 \mathrm{p}$ & B & B1 & $X$ & B & B5 & B2 & B2 & B & $X$ \\
\hline $\mathrm{ks} 20^{\prime \prime}$ & B & B1 & $X$ & B & B3 & B2 & B1 & B & B \\
\hline kat $1^{\prime \prime}$ & B & B1 & $X$ & B & B5 & B2 & B2 & B & $X$ \\
\hline Jit1 & B & B1 & $X$ & B & B5 & B2 & B2 & B & $X$ \\
\hline eit1b2 & B & B1 & $X$ & B & B5 & B2 & B2 & B & $X$ \\
\hline $\mathrm{kdb} 2^{\prime}$ & B & B1 & $X$ & B & B4 & B2 & B2 & B & B \\
\hline kit1" & B & B2 & B & B & B5 & B2 & B1 & B & $X$ \\
\hline Jat1 & B & B2 & B & B & B5 & B2 & B1 & B & $X$ \\
\hline
\end{tabular}

Columns belong to the variables, and rows belong to the parameters of the cell cycle model. Names of the variables and parameters can be checked in the previous papers related to this model [3,5].

Identical labels (except for X) within a column indicate globally similar sensitivity functions. Sign X shows that no similar sensitivity function was found for the same variable.

Sensitivity functions of parameters $k i 2 O^{\prime}, k i 2 O^{\prime \prime}, k s s p n, k d s p n, k s n 2^{\prime}, k i t 1^{\prime}, k s b u d, k d b u d, J_{s p n}, e b u d b 5$, eorib2, kdori, ksori, kdib5, and kdib2 were near zero at all times and for all variables. The rows corresponding to these parameters are not given in the table. 


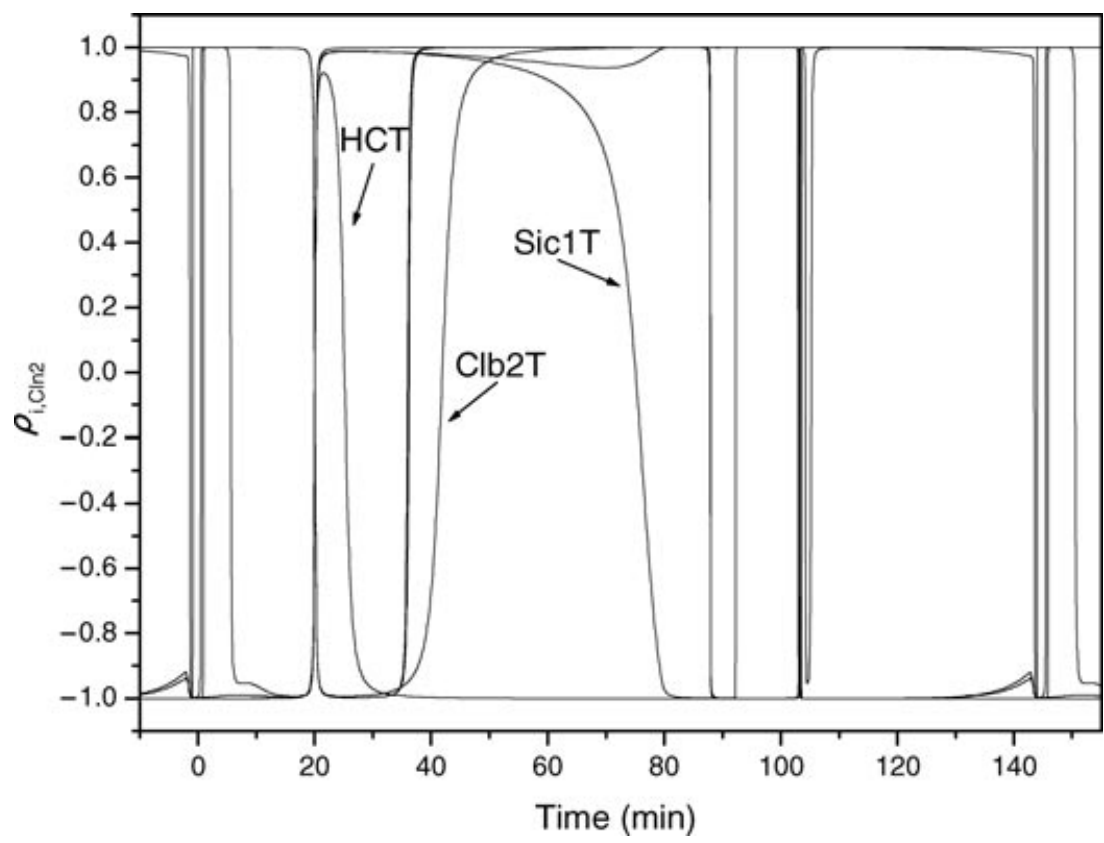

Figure 5 Correlation of the sensitivity vector of $C \ln 2$ with the sensitivity vectors of all other variables, considering parameters kasbf, kisbf', esbfn3, BCK0; CLN3MAX, Dn3, and Jn3. Measure $\cos \theta$ is close to \pm 1 at most times and for all variables, indicating local similarity of these vectors.

If two cells in Table I belonging to the same variable (or column) have identical labels, it means that the change of the corresponding parameters has a similar effect for this variable during the whole cycle. This means that the effect of changing one parameter can be compensated by the appropriate change of the other parameter. If two parameters belong to the same local similarity group for a given pairs of variables, then this compensation extends to the concentration-time curve belonging to the other variable.

Figure 6 shows the concentration-time curve of $C \ln 2$ in the original model during a cycle. If parameter

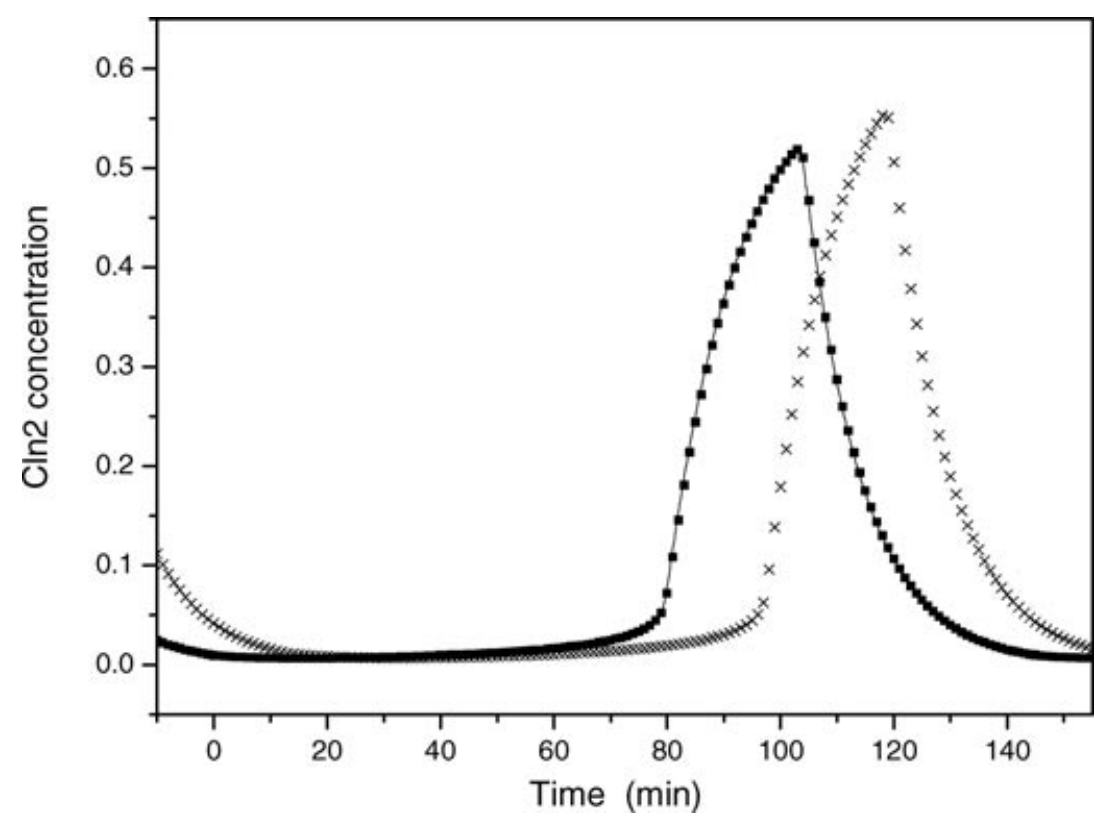

Figure 6 The concentration of $C \ln 2$ in the original model (solid line), the calculated $C \ln 2$ concentration when parameter $k_{i s b f^{\prime}}$ is increased by $10 \%$ (crosses), and when parameters kisbf and BCKO are simultaneously increased by $10 \%$ and $22 \%$, respectively (dots). 


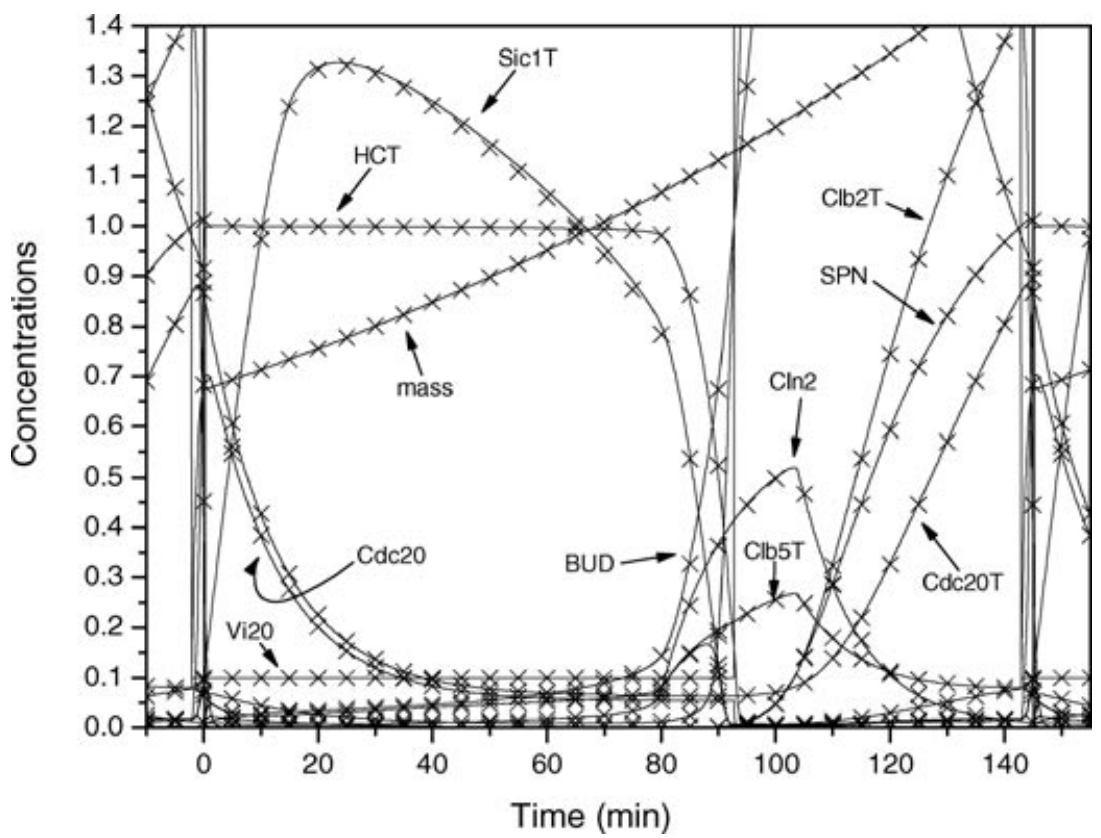

Figure 7 The concentration-time curves of all species of the cell cycle model, calculated by the original parameter set (solid line) and when two important parameters were simultaneously changed (crosses). In the later case parameters kisbf and $B C K O$ were increased by $10 \%$ and $22 \%$, respectively.

$k i s b f^{\prime}$ is increased by $10 \%$, this curve changes significantly. However, an appropriate $(+22 \%)$ change of another parameter $(B C K 0)$ perfectly restores the original curve. The reason is that the sensitivity function curves of $C l n 2$ with respect to parameters kisbf and $B C K O$ show global similarity; therefore, the parameter changes have similar effect anytime during the cell cycle. As Fig. 5 shows, the sensitivity coefficients of all variables belonging to these parameters also exhibit local similarity; therefore, the simultaneous change of parameters kisbf and $B C K O$ results in the same concentration-time curves for all species (see Fig. 7) compared to the original model.

\section{SIGNIFICANCE OF THE SIMILARITY OF THE SENSITIVITY FUNCTIONS OF THE CELL CYCLE MODEL}

Global similarity of sensitivity functions in biochemical and biological models is of high importance, because it can be related to the robustness of living organisms. In general, in nonlinear systems the change of each parameter has different effect on the simulated time curves of variables. Change of one parameter can be fully compensated by changing another parameter only in special cases, when, for example, only the ratio of two parameters influences the results. However, if the sensitivity functions are globally similar, then the change of these parameters can compensate each other. Most parameters in the cell cycle model are related to enzyme activities. These enzyme activity parameters can change due to mutations. If such a mutation is not lethal, but harms the normal operation of the cell, a further mutation, influencing another parameter in the same similarity group, can fully restore the normal operation. Since the requirement is not the (unlikely) restoration of the value of the same parameter, this mending mechanism is much more versatile.

\section{CONCLUSIONS}

Models can be effectively analyzed via perturbations. Local parametric sensitivity coefficients $\partial Y_{i} / \partial p_{k}$ show how the calculated variable value $Y_{i}$ changes due to small change of parameter $p_{k}$. Timescale and sensitivity analyses are frequently applied tools for the investigation of chemical kinetic models, and these tools are well applicable for any other model based on differential equations. Inspection of the eigenvalues of the Jacobian reveals the actual timescales of the system, and also excitation and relaxation periods can be distinguished. The dynamical dimension of the system can also be calculated $[19,25,26]$. This dynamical dimension is equal to the number of differential equations in a minimal model of the same accuracy. 
Zsély et al. [16-19] have investigated a series of combustion models, looking for the similarity features of local sensitivity functions. Two sensitivity functions may have identical shapes, which means that one can be obtained from the other by multiplying it with a constant. These sensitivity functions are globally similar, and in this case the changes of the related parameters have similar effect for the value of a variable during the whole time interval. If the shapes of the sensitivity functions for two parameters are identical for all variables, then any change of one of the parameters can be compensated by changing the other parameter.

In the combustion models investigated so far, the nonzero sensitivity functions were either all globally similar or global similarity was not detected. The similarity of sensitivity functions was related to the existence of low-dimensional slow manifolds in chemical kinetic systems and the presence of an autocatalytic runaway during a period. Until now, all models showing the similarity of local sensitivity functions described high-temperature gas kinetics (combustion) systems.

The dynamical dimension of the cell cycle model of Chen et al. [3] was also found to be much smaller than the number of variables and excitation (autocatalytic) periods were identified [5]. Therefore, similarity of the sensitivity functions was expected. Numerical calculation and investigation of the local sensitivity functions of this model showed that the sensitivity functions are really similar, but a qualitatively new feature of these sensitivity functions was found. For each variable, most of the sensitivity functions were globally similar, but these functions could be sorted to several groups of global similarity. Such a feature was not found in the previously investigated chemical kinetic systems.

A possible reason for this new type of global similarity can be related to the basically different nature of the cell cycle model compared to the combustion systems. In high-temperature gas phase combustion models, the autocatalytic runaway is caused by a single group of strongly coupled reaction steps, the chainbranching $\mathrm{H} / \mathrm{O}$ radical reactions. In the budding yeast cell cycle, several loosely connected excitation centers are present, composed by enzyme catalytic groups of reactions, and these are firing one after the other according to a strict order [5]. This may cause the multiple global similarities of the sensitivity functions. Of course, this is a qualitative explanation, which should be extended to a formal mathematical proof.

If two sensitivity functions of variable $i$ have similar shapes, it means that the perturbation of the corresponding parameters have similar effects, which is an important structural information. Global similarity of the sensitivity functions of two parameters means that the change of one parameter can be fully compensated by an appropriate change of the other parameter. This is important information for the validation of complex dynamical models.

The joint application of local sensitivity and timescale analyses is a very powerful set of tools for the investigation of complex dynamical models. It reveals the excitation periods, the change of dynamic dimension, and the effect of parameter perturbation. Similarities of local sensitivity functions carry important information on the role of parameters in the model.

\section{BIBLIOGRAPHY}

1. Tyson, J. J.; Novák, B.; Chen, K. C.; Val, J. In Progress in Cell Cycle Research; Meijer, L.; Guidet, S., Tung, H. Y. L. (Ed.); Plenum Press: New York, 1995; pp. 1-8.

2. Novák, B.; Csikász-Nagy, A.; Győrffy, B.; Nasmyth, K.; Tyson, J. J. Philos Trans R Soc London B 1998, 353, 2063-2076.

3. Chen, K. C.; Csikász-Nagy, A.; Györffy, B.; Val, J.; Novák, B.; Tyson, J. J. Mol Biol Cell 2000, 11, 369391.

4. Chen, K. C.; Calzone, L.; Csikász-Nagy, A.; Cross, F. R.; Novák, B.; Tyson, J. J. Mol Biol Cell 2004, 15, 3841-3862.

5. Lovrics, A.; Csikász-Nagy, A.; Zsély, I. Gy.; Zádor, J.; Turányi, T.; Novák, B. BMC Bioinformatics 2006, 7 , 494.

6. Saltelli, A.; Scott, E. M.; Chen, K. (Eds.). Sensitivity Analysis; Wiley: Chichester, UK, 2000.

7. Saltelli, A.; Tarantola, S.; Campolongo, F.; Ratto, M. Sensitivity Analysis in Practice. A Guide to Assessing Scientific Models; Wiley: New York, 2004.

8. Turányi, T. J Math Chem 1990, 5, 203-248.

9. Tomlin, A. S.; Turányi, T.; Pilling, M. J. In Low Temperature Combustion and Autoignition, Pilling, M. J.; Hancock, G. (Eds.); Elsevier: Amsterdam, 1997; pp. 293-437.

10. Ihekwaba, A. E.; Broomhead, D. S.; Grimley, R. L.; Benson, N.; Kell, D. B. Syst Biol (Stevenage) 2004, 1, 93-103.

11. Lüdtke, N.; Panzeri, S.; Brown, M.; Broomhead, D. S.; Knowles, J.; Montemurro, M. A.; Kell, D. B. J R Soc Interface 2008, 5, 223-235.

12. Yue, H.; Brown, M.; He, F.; Jia, J.; Kell, D. B. Int J Chem Kinet 2008, 40, 730-741.

13. Reuven, Y.; Smooke, M. D.; Rabitz, H. J Comput Phys 1986, 64, 27-55.

14. Rabitz, H.; Smooke, M. D. J Phys Chem 1988, 92, 1110 1119.

15. Vajda, S.; Rabitz, H. Chem Eng Sci 1992, 47, $1063-$ 1078.

16. Zsély, I. Gy.; Zádor, J.; Turányi, T. J Phys Chem A 2003, 107, 2216-2238.

17. Zsély, I. Gy.; Turányi, T. Phys Chem Chem Phys 2003, 5, 3622-3631. 
18. Zádor, J.; Zsély, I. Gy.; Turányi, T. Int J Chem Kinet 2004, 36, 238-252.

19. Zsély, I. Gy.; Zádor, J.; Turányi, T. Combust Theory Modell 2005, 9, 721-738.

20. Caracotsios, M.; Stewart, W. E. Comput Chem Eng 1985, 9, 359-365.

21. Lutz, A. E.; Kee, R. J.; Miller, J. A. SENKIN: A FORTRAN program for predicting homogeneous gas phase chemical kinetics with sensitivity analysis; Sandia National Laboratories Report SAND87-8248, 1988.

22. Kee, R. J.; Rupley, F. M.; Miller, J. A. CHEMKINII: A FORTRAN chemical kinetics package for the analysis of gas-phase chemical kinetics; Sandia report No. SAND79-8009B, 1989.

23. Everitt, B. S.; Landau, S.; Leese, M. Cluster Analysis. Oxford University Press: New York; 2001.

24. Glenn, W. Milligan Code HCINFLU: Generalized Agglomerative Hierarchical Clustering Algorithms; available at http://www.pitt.edu/ csna/Milligan/readme.hc, accessed 2007.

25. Tomlin, A. S.; Whitehouse, L.; Lowe, R.; Pilling, M. J. Faraday Discuss 2001, 120, 125-146.

26. Büki, A.; Perger, T.; Turányi, T.; Maas, U. J Math Chem 2002, 31, 345-362. 